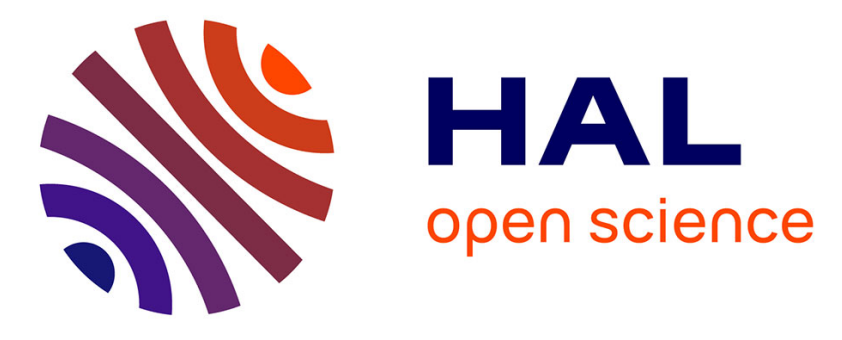

\title{
Heterogeneous multipath networks: Flow vs Packet based routing in a size-aware context
}

Elie Bouttier, Riadh Dhaou, Fabrice Arnal, Cédric Baudoin, Emmanuel Dubois, André-Luc Beylot

\section{- To cite this version:}

Elie Bouttier, Riadh Dhaou, Fabrice Arnal, Cédric Baudoin, Emmanuel Dubois, et al.. Heterogeneous multipath networks: Flow vs Packet based routing in a size-aware context. IEEE Global Communications Conference "Global Hub: Connecting East and West" (GLOBECOM 2017), Institute of Electrical and Electronics Engineers ( IEEE); IEEE Communications Society (IEEE ComSoc), Dec 2017, city-state, Singapore. pp.1-6, 10.1109/GLOCOM.2017.8255095 . hal-03116301

\section{HAL Id: hal-03116301 https://hal.science/hal-03116301}

Submitted on 21 Jan 2021

HAL is a multi-disciplinary open access archive for the deposit and dissemination of scientific research documents, whether they are published or not. The documents may come from teaching and research institutions in France or abroad, or from public or private research centers.
L'archive ouverte pluridisciplinaire HAL, est destinée au dépôt et à la diffusion de documents scientifiques de niveau recherche, publiés ou non, émanant des établissements d'enseignement et de recherche français ou étrangers, des laboratoires publics ou privés. 


\title{
Heterogeneous multipath networks: Flow vs Packet based routing in a size-aware context
}

\author{
${ }^{1}$ Élie Bouttier, ${ }^{2}$ Riadh Dhaou, ${ }^{3}$ Fabrice Arnal,,${ }^{3}$ Cédric Baudoin, ${ }^{4}$ Emmanuel Dubois, ${ }^{2}$ André-Luc Beylot \\ ${ }^{1}$ elie.bouttier@enseeiht.fr, TéSA, Université de Toulouse; F-31071 Toulouse, France \\ $2\{$ dhaou,beylot\}@enseeiht.fr, IRIT, Université de Toulouse; F-31071 Toulouse, France \\ ${ }^{3}$ \{fabrice.arnal,cedric.baudoin\}@thalesaleniaspace.com, Thales Alenia Space \\ ${ }^{4}$ emmanuel.dubois@cnes.fr, Centre National d’Études Spatiales
}

\begin{abstract}
We are facing a steady increase in Internet usages and bandwidth requirements. However, many terrestrial infrastructures remain unchanged due to exorbitant modernization costs, particularly in rural areas. In front of the aging of their infrastructures, concerned users are turning towards satellite internet access. Indeed, these solutions offer a high-throughput internet access at a moderate cost of deployment. Unfortunately, the long delay introduced by GEO satellite degrades significantly the user Quality of Experience (QoE) in many cases.

In this paper, we consider a hybrid access, composed of a low data rate terrestrial path and a satellite path, within a Content Delivery Network (CDN) context. Thanks to the CDN, requested content size is known allowing to use this information in routing schemes to maximize the users' QoE. Then, we compare flow and packet based routing in this heterogeneous and size-aware context. We finally conclude on the limited interest in packetbased routing compared to flow-based routing, privileged by its simplicity and good performance from a $\mathrm{QoE}$ point of view.
\end{abstract}

\section{INTRODUCTION}

Internet usage is growing very fast, leading to an exponential bandwidth increase. Thereby, internet infrastructures need to be upgraded to face these new requirements. While there is a massive deployment of fiber (FTTH) in some cities, other areas do not evolve. That is the case for rural areas where modernization costs are prohibitive given potential customers. Hence these areas become underserved regarding new bandwidth standards.

Satellite networks allow bringing a high bandwidth internet access at a moderate cost of deployment which provides users with new services they could not or hardly use before like video on demand ( VoD), video streaming, geolocation services, photo gallery, etc. However, satellite access suffers from the significant delay introduced by the signal propagation between the Earth and the geostationary orbit leading to a notable negative impact on user Quality of Experience (QoE). Interactive services, like video games or web traffic, suffer particularly badly from this long delay. From this perspective, a hybrid network associated with a smart routing policy may provide better QoE for end users by using the best of both networks.

Several techniques exist, in the literature, to efficiently exploit the diversity of paths. Indeed, multipath networks provide among others a better throughput, robustness to congestion and resilience to breakdown. They can be classified into two classes, flow-based routing schemes, and packetbased routing schemes, the first class being a subset of the second one. Packet-based routing offers new possibilities compared to flow-based routing. For instance, it allows to aggregate the capacity of different paths for the same flow or to balance the load between several paths at a finer scale.

However, heterogeneous networks bring specific issues. Indeed, differences in speed, latency or packet loss reveal several problems to exploit the different links properly. Especially, packet-based routing schemes, associated to paths with differents delays, worsen out-of-order delivery of packets, which is misunderstood by TCP congestion algorithm. Thereby, packet-based routing in heterogeneous environments needs specific mechanisms to work properly.

To avoid the bad effect of out-of-order packets, different solutions have been proposed like reordering buffer at the end side of the heterogeneous networks [1] or to voluntarily send packets in disorder in a way to receive them in order [2]. Some works [3] try to improve TCP reordering robustness to make it suitable for heterogeneous networks. The new transport protocol Multipath-TCP (MP-TCP) [4] can effectively aggregate path throughputs by using separate TCP sub-flows for each path.

These proposals try to maximize different metrics like link occupancy or aggregate capacity. However, the lack of relevant flow information leads to bad routing decision, especially by sending delay sensitive data on the satellite path, which significantly reduces the end-user QoE. In this paper, we consider a Content Delivery Network (CDN) context. The studied architecture, presented in II, allows accessing the content information, in particular, the flow size. Furthermore, the managed context allows deploying specific hybrid routing mechanisms. In this size-aware context, we aim to study the interest from a QoE point of view of packet-based routing compared to flow-based routing.

After the introduction of the architecture, we present the MinFCT flow-based and size-aware routing scheme introduced in [5]. Section IV exhibits different packet-based routing schemes, namely size-aware path aggregation as well as severals complementary mechanisms which aim to improve 
flow-based routing. Then, we describe the implementation of a simulation framework, to evaluate the performance of the proposed routing schemes. Finally, we conclude on pros and cons of flow-based and packet-based routing schemes in our size-aware and heterogeneous context.

\section{ARChitecture}

\section{A. Overall architecture}

Studied routing schemes operate on a multipath architecture represented in figure 1. This architecture allows a home network to access the Internet through a multipath network composed of a terrestrial and a satellite path. These paths are supposed to be the bottlenecks of the network. The multipath network is operated by a virtual Internet Service Provider (ISP) which manages it through two gateways located on each side and which operate as Layer 7 proxies. We reuse the terminology introduced in [6] with the Intelligent User Gateway (IUG) located on the user side and the Intelligent Network Gateway (ING) located on the network side.

The virtual operator also manages its telco Content Delivery Network (CDN) interconnected with several upstream CDNs through Content Delivery Networks Interconnection (CDNI) protocols [7]. Thereby, the hybrid operator can access flow information, especially the size, for contents hosted on these upstream CDNs by different Content Providers. CDNI provides a specific interface to get this kind of information called the Metadata Interface (MI) [8].

Each gateway has to choose on which path each flow should be routed. In flow-based routing schemes, each flow is assigned to only one path. Conversely, in packet-based routing schemes, each flow can be routed on both paths simultaneously and/or reassigned from one path to another. We do not consider here the protocol aspects of the architecture, which will be subject of further works.

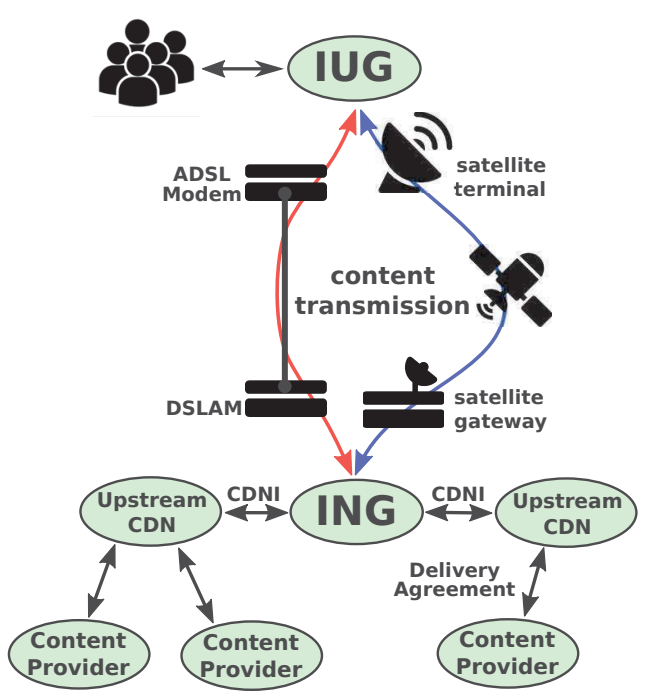

Fig. 1. Studied architecture.

\section{B. Scheduling choices}

Although the Shortest Remaining Processing Time (SRPT) discipline is known to give the best performance in a G/G/1 queue [9] and so traditional networks, it is seldom used as it is unfair and causes large flows starvation. To ensure fairness in our multipath context, concurrent flows on the same path are served accordingly to a Fair Flow (FF) scheduler which attempts to emulate the fairness of the Processor Sharing (PS) model. This scheduler ensures an asymptotic equal throughput between flows assigned to the same link. As well as ensuring fairness, this choice allows observing routing schemes performance in a way decorrelated from scheduling effects.

The Fair Flow scheduler is an adaptation of the Fair Queueing (FQ) scheduler [10] [11] which was designed for packet switched router. It is based on the concept of virtual time. This quantity corresponds to the assumed last service time of each flow, if the flows are fairly served. The virtual time is initialized to the flow arrival time. Every time a link is available, the flow to be served is selected according to the following formula:

$$
\underset{i}{\operatorname{argmin}}\left\{\text { virtualtime }_{i}+n_{l} \cdot \frac{\min \left(r_{i}, \text { payload }\right)}{c_{l}}\right\}
$$

with $n_{l}$ the number of flows currently assigned to the link $l, r_{i}$ the remaining amount of bytes of flow $i, c_{l}$ the capacity of the link $l$ and payload being the maximum payload size of a packet. The served flow virtual time is then updated as follows:

$$
\text { virtualtime }_{i} \leftarrow \text { virtualtime }_{i}+n_{l} \cdot \frac{\min \left(r_{i}, \text { payload }\right)}{c_{l}}
$$

\section{Flow-BASED ROUTING SCHEME: MinFCT}

For the remainder of this paper, we compare packet-based routing schemes to the flow-based size-aware MinFCT routing scheme presented in [5]. This scheme aims to minimize the average Flow Completion Time (FCT) by choosing the best path for each given flow. The following formula, which takes the content size into account, gives the path to which the flow should be assigned:

$$
\underset{i}{\operatorname{argmin}}\left\{d_{i}+\left(n_{i}+1\right) \cdot \frac{s}{c_{i}}+\sum_{k=0}^{n_{i}} \frac{\min \left(r_{k}, s\right)}{c_{i}}\right\}
$$

It consists in estimating, for each path $i$, the completion time of the considered flow of size $s$ and chooses the path which minimizes this quantity. The flow completion time is assessed from the delay $d_{i}$ and capacity $c_{i}$ of the path, known or estimated from Management Information Base (MIB), TCP-stack or Throughput Guidance [12] for instance, and the number $n_{i}$ of flows already assigned to the path $i . r_{k}$ is the remaining amount of data of flow $k$. 
The transmission time estimation supposes that each flow receive an equal part of the overall available capacity. However, the number of flows sharing a path varies over time, according to flow arrivals and departures. While departures can be predicted thanks to the knowledge of flows size, arrivals remain unpredictable. Thus, we observed that, instead of predicting flow departures, considering a constant number of competing flows to estimate the Flow Completion Time gives good results.

The fact to assign a flow to a path will slow down other flows already assigned to the same path. This is taken into account by adding, for each flow $k$ assigned to the path $i$, an additional transmission time $\frac{\min \left(r_{k}, s\right)}{c_{i}}$ where $r_{k}$ is the remaining amount of data to be transferred before the flow $k$ ends.

\section{PACKET-BASED ROUTING SCHEMES}

In this section, we present different routing mechanisms which come out of the flow-based class. This means different packets belonging to the same flow may be routed through different paths.

\section{A. Size-aware aggregation}

Aggregation consists in splitting up, for a given flow, the set of packets on both paths, allowing to obtain an increased throughput for the given flow. Although this is beneficial for large flows, short flows suffer from the large satellite propagation delay which may prove to be greater than the flow completion time on the terrestrial path only. For a given flow $i$, the aggregation with the satellite network is useful only if the remaining amount of data to transfer $r_{i}$ verifies the following inequality:

$$
n \cdot \frac{r_{i}}{c_{t}}+d_{t}>d_{s}
$$

where $n$ is the number of flows, $c_{t}$ the capacity of the terrestrial path and $d_{t}$ (resp. $d_{s}$ ) the propagation delay of the terrestrial path (resp. of the satellite path). Thereby, the size knowledge prevents performance degradation due to the satellite delay. Let's notice that a large flow can be, first, aggregated on both paths until the remaining amount of data becomes small enough to invalidate the previous inequality. This mechanism allows to synchronize the reception of last bytes on both paths. We call this behavior Tail Sync, and the Aggregation Threshold designates the minimum amount of remaining data necessary to trigger satellite aggregation (which depends on the number of transmitting flows $n$ ).

However, we do not expect significant improvements from the aggregation compared to the flow-based routing, as soon as several flows are in competition. Indeed, the terrestrial bandwidth is precious as it is not subject to the long satellite propagation time. Thereby, sending packets of the same flow on both paths is a waste of terrestrial bandwidth because it could be used beneficially to others short flows that we would send on the terrestrial path only. As the terrestrial capacity is lower than the satellite one, the aggregation benefit for large flows does not worth the potential bandwidth savings for short flows.

\section{B. Idle Link Opportunistic Aggregation (ILOA)}

Although aggregation is susceptible to degrade the performance with concurrent flows, it can still be used positively under low load. Thereby, the Idle Link Opportunistic Aggregation (ILOA) mechanism aims to be used together with a flow-based routing scheme, for instance MinFCT, and consists in allowing a path to be used for aggregation only if there are no flows currently assigned to this path (idle link). In this case, the served flow is chosen between the flows assigned to other links accordingly to the Fair Flow scheduler. Here again, the size-aware condition on satellite aggregation is used to avoid the degradation of performance due to the large propagation time. This mechanism should allow a single flow to benefit from multiple paths capacities without degrading the performance of the competing flows.

\section{Reassignment}

The reassignment mechanism is also associated with a flowbased routing scheme like MinFCT. It consists in forgetting all flows assignment and re-playing the allocation scheme according to a given policy discussed in the following. The objective is to favor short flows to be assigned to the terrestrial networks. Therefore, the allocation scheme is replayed on the flows, sorted from the flow having the least remaining bytes to send to the one having the most remaining bytes.

This mechanism is expected to improve the performance by relocating some flows, as networks conditions evolve, to stay close to the optimal allocation. Thereby, some flows can be reassigned from the terrestrial to the satellite path as the terrestrial network becomes congested, for example, due to a burst of short flows. Conversely, some flows can be reassigned from satellite to terrestrial path as this last one becomes available due to previous flow ends.

We consider two possibilities to choose reassignment moments:

- on each flow arrival or flow completion

- periodically, with a given period

Arrival and departure epochs seem to be the best moments to trigger the reassignment mechanism as they provoke an evolution of the situation. However, these moments can be very close under high traffic networks condition, which can lead to computational issues. Periodic reassignment is a way to make the implementation of the reassignment mechanism scalable so that we will only focus on it.

This mechanism needs to slightly modify the MinFCT allocation scheme. First, we no longer consider the initial flow size but naturally the remaining amount of data to be sent. Secondly, we need to avoid reallocation from satellite to terrestrial path when the benefit from reallocation is smaller than the time for packets in flight on the satellite link to arrive. Thereby, we save terrestrial bandwidth for other short flows. This is accomplished by memorizing the last packet sending time on each link. With this information and an 
estimation of the path latency, the remaining flight time can be estimated and compared to the estimated flow completion time as computed in the regular MinFCT scheme. We refer to this mechanism as FlightTime.

\section{EXPERIMENTATION}

Finally, we implemented a simulation environment in order to evaluate the performance of proposed routing mechanisms. The simulator framework is composed of a test client which retrieves HTTP objects from a content server through both Intelligent Gateways using SOCKS [13]. As the IUG always send the content request through the terrestrial path, the ING implements the evaluated routing schemes. The test client implements a pool of users. Each user follows the given cycle:

1) waiting for a random time;

2) sending a request for a given content;

3) waiting for the reception of the last byte of the content.

We observe the Flow Completion Time (FCT), which is the elapsed time between the sending of the request and the reception of the last part of the content. This metric is used to evaluate the end-user Quality of Experience, considering that short flows are more delay sensitive (e.g. web traffic) than large flows (e.g. video streaming).

The average waiting time is 1 second. The size of requested contents is equal to a multiple of the payload of a packet, the number of packets follows a Zipf law. The law is shifted to ensure a minimum flow length of 7 packets and truncated to a maximum flow length of 6000 packets; the Zipf law parameter $\alpha$ is 1.8 . Packets have a payload of 1450 bytes with a header of 50 bytes. Experiments are done using UDP with perfect links (no losses) between Intelligent Gateways, which avoid the side effects of a reliable transport protocol. The terrestrial path has a capacity of $2 \mathrm{Mbits} / \mathrm{s}$ with a $20 \mathrm{~ms}$ delay while the satellite path has a capacity of $8 \mathrm{Mbits} / \mathrm{s}$ with a $300 \mathrm{~ms}$ delay.

\section{A. Size-aware aggregation}

Figure 2 shows a comparison in a size-aware context between flow-based routing with MinFCT and packet-based routing with an aggregation mechanism. The curves represent the average Flow Completion Time for different number of users in the system. In most situations, the aggregation significantly deteriorates the performance, confirming our assumption that aggregation is a bad idea as soon as there are several competing flows.

For very few users, the aggregation scheme can slightly outerperform MinFCT due to the aggregation of terrestrial and satellite bandwidths for large flows. However, the observed gain is low, among others due to the large difference between the terrestrial and the satellite capacities which limits the benefit of the aggregation. Moreover, only large flows which exceed the Aggregation Threshold can benefit from aggregation but they are few. Indeed, the flow size follows a Zipf which is characterized by a long-tailed distribution, explaining the low gain on average.

We can compare in figure 3 the terrestrial and satellite link occupancy for the MinFCT and the size-aware aggregation

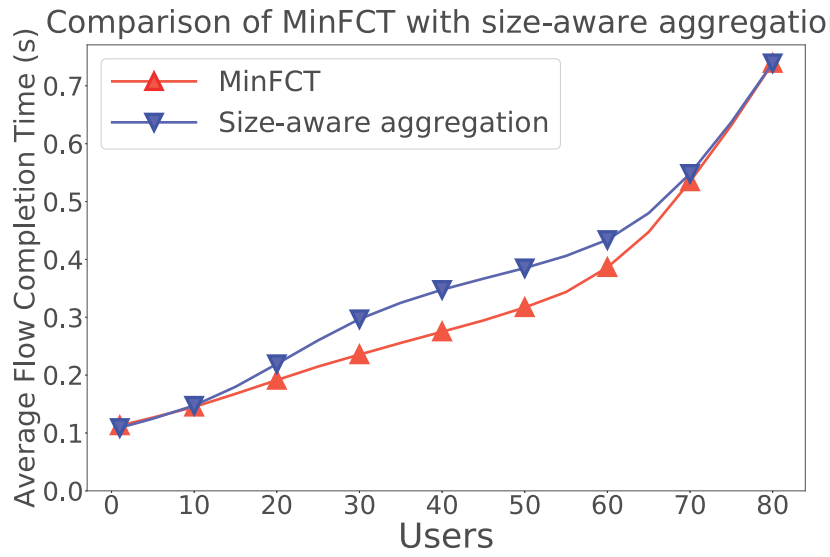

Fig. 2. Comparison of MinFCT and size-aware aggregation. The flowbased MinFCT scheme shows a better average Flow Completion Time than aggregation.

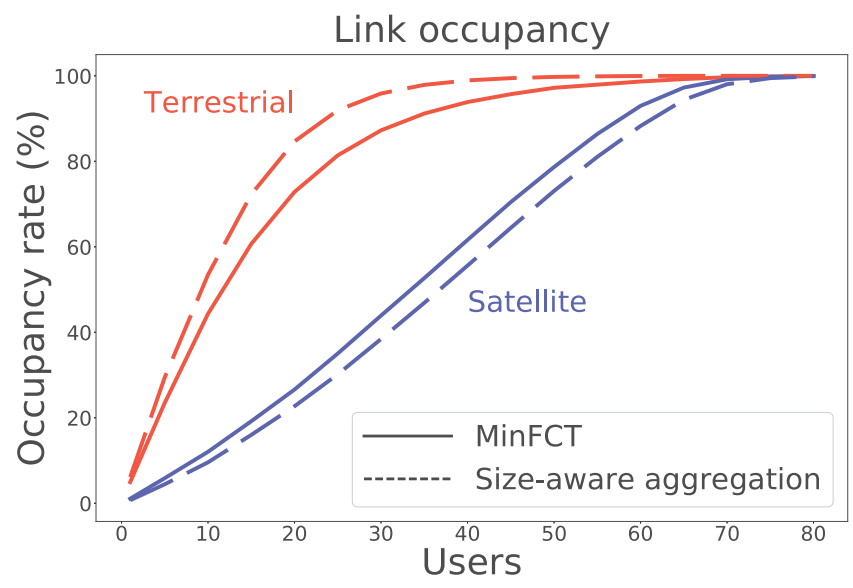

Fig. 3. Comparison of links occupancy under MinFCT and size-aware aggregation. The flow-based routing scheme MinFCT shows an ability to reduce the terrestrial path usage which is beneficial for performance as seen in figure 2.

routing schemes. We observe that the terrestrial path is less busy under MinFCT routing scheme compared to the aggregation. In fact, the terrestrial path is reserved for short flows, allowing to obtain better performance as observed in figure 2 .

The size-aware aggregation performance, in blue in figure 2 , shows two points of inflection corresponding, first, to the saturation of the terrestrial path, then, to the saturation of the satellite path, which can be verified on figure 3. The MinFCT routing scheme avoids the first inflection point by preventing the terrestrial congestion. This is another explanation of the better results obtained with MinFCT.

\section{B. Idle link opportunistic aggregation}

As link aggregation is beneficial under low load but harmful under high load, the Idle Link Opportunistic Aggregation (ILOA) mechanism allows aggregation only on idle link. Figure 4 shows a comparison of the average FCT under MinFCT with and without opportunistic aggregation. We observe that 


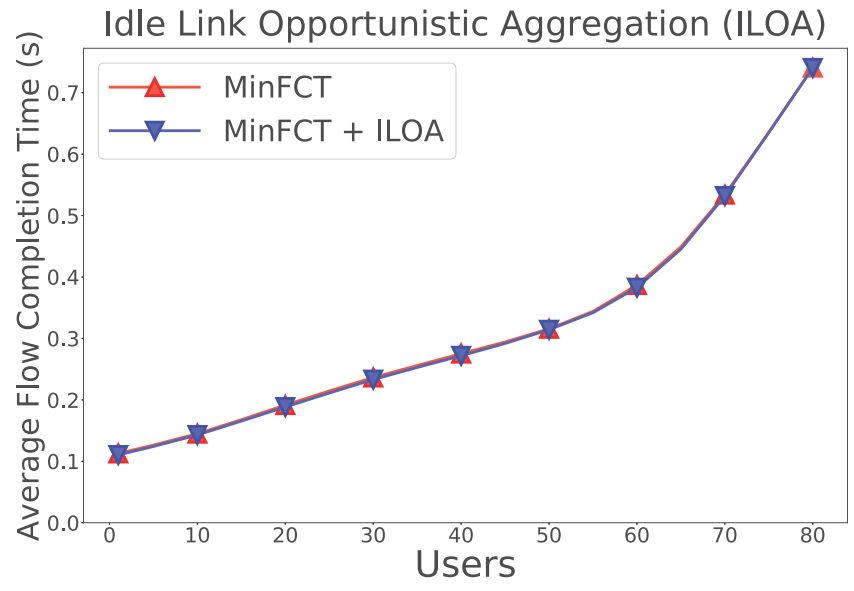

Fig. 4. Comparison of MinFCT with and without Idle Link Opportunistic Aggregation. The opportunistic aggregation mechanism fully mitigates the performance degradation obtained with size-aware aggregation. ILOA even allows to improve MinFCT performance but the gain is very small due to the chosen law and the large difference in path capacities.

Idle Link Opportunistic Aggregation (ILOA)

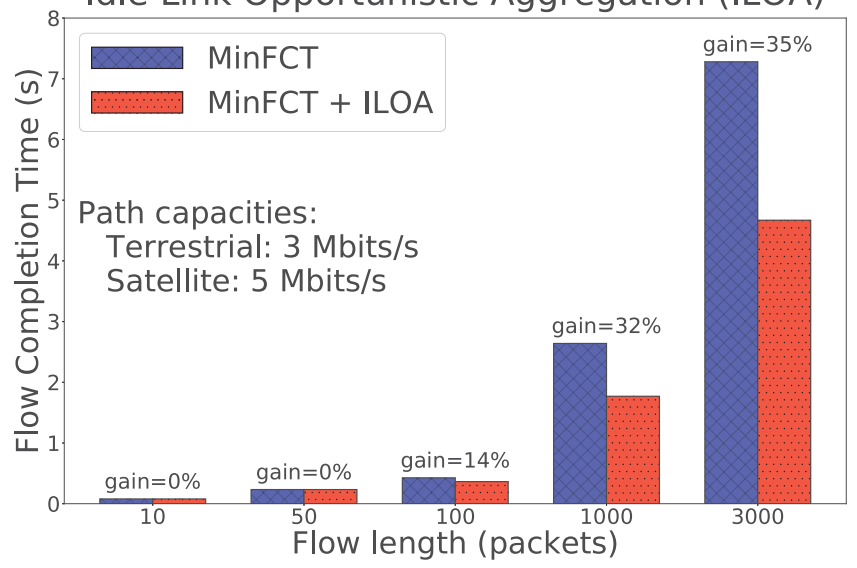

Fig. 5. Gain of ILOA mechanism for different flow lengths. For short flows which do not exceed the Aggregation Threshold, the gain is zero. For large flows, the gain is up to $35 \%$.

MinFCT with opportunistic aggregation slightly improves the performance compared to standard MinFCT, as size-aware aggregation in situations with few users only. However, this gain is present for a large number of users whereas sizeaware aggregation deteriorated the performance. The benefit remains low for the same reasons as stated for size-aware aggregation, namely the large difference in path capacities and the chosen law, which limits the number of large flows. Nonetheless, for closer path capacities or considering an advantageous distribution in large flows, we can expect a better improvement.

Figure 5 represents the gain of the ILOA mechanism under the MinFCT assignment scheme for different flow lengths. Here, there are no concurrent flows so link aggregation can be fully exploited. Path capacities are, here, closer compared to the others experiments. For flows shorter than the Aggre-

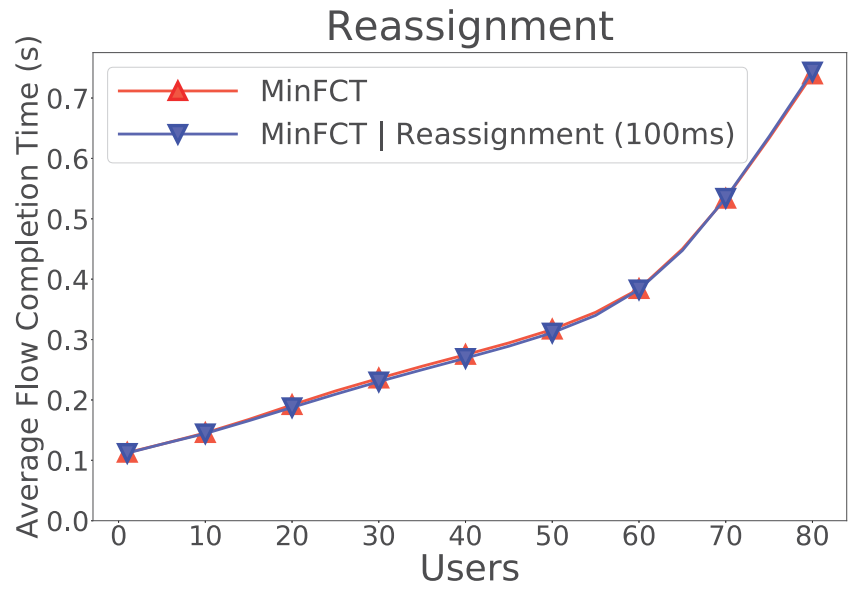

Fig. 6. Gain brought by reassignment using a $100 \mathrm{~ms}$ reassingment period Reassignment only shows a small performance improvement.

gation Threshold, the gain is obviously zero as the flow is fully sent on the terrestrial path. For flows larger than the Aggregation Threshold, the gain increases with the flow length as the transmission time becomes predominant in front of the propagation time. The gain is up to $35 \%$, close to the optimal, which is $37.5 \%$ for given path capacities. For the path capacities usually used in the others experiments $-2 \mathrm{Mbits} / \mathrm{s}$ vs. $8 \mathrm{Mbits} / \mathrm{s}$ - the gain is up to $20 \%$ for large flows. As stated before, the expected average gain is strongly dependant of the distribution of short and large flows.

\section{Reassignment}

Figure 6 shows a comparison of performance obtained with and without reassignment using the MinFCT allocation scheme. The results only show a little improvement in favor of the reassignment mechanism. The experiment is conducted using a $100 \mathrm{~ms}$ reassignment period; others period have been tested without observing a better improvement. The reasignment mechanism is also reproducing the Tail Sync behavior: when the remaining amount of data to be sent of a large flow becomes smaller than the Aggregation Threshold, the flow becomes relocated on the terrestrial path. However, the benefit of this reassignment is equal to the loss inflicted to terrestrial flows - current or future - future ones not being taken into account in the reassignment decision. This is part of the explanation of the low improvement brought by this mechanism. While experiments have been realised with a constant law shape and constant path capacities, we can expect a better improvement in a more dynamic environment. As the ILOA mechanism, the gain is strongly dependent of flow size distribution, particularly the distribution of medium flow. Indeed, these are more likely be reassigned from one path to another compared to short or long flows for which the routing decision is unlikely to evolve.

The FlightTime mechanism should prevent reassignment from satellite to terrestrial path if the gain of the operation is void in the light of the need to wait for in-flight packets 


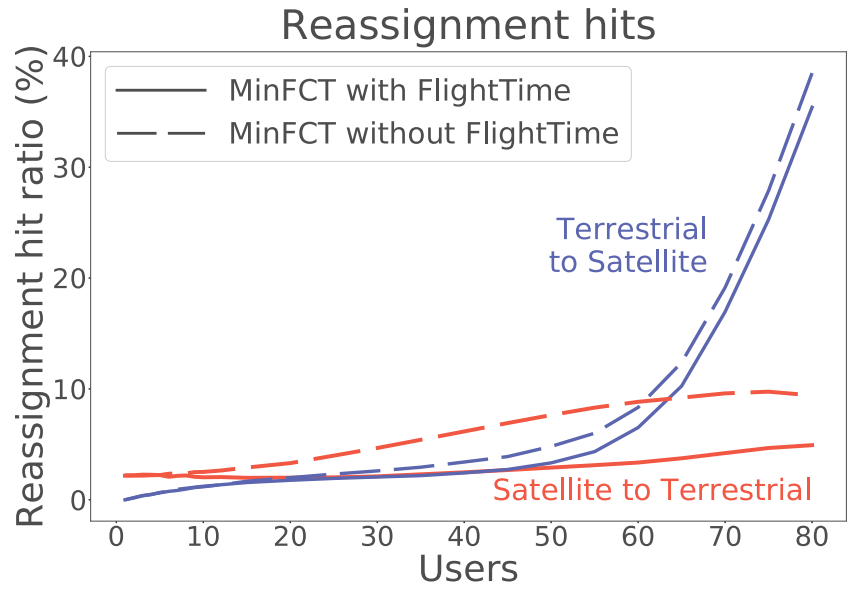

Fig. 7. Comparison of the percentage of flows subject to reassignment for MinFCT and MinFCT without FlightTime prevention. Path statistics are established on first reassignment only. We observe that taking the FlightTime into account allow to significantly reduce the number of reallocations, especially from satellite to terrestrial path.

on the satellite path. Figure 7 allows observing the effect of the FlightTime mechanism. As expected, the reassignment hit ratio from satellite to terrestrial path is significantly reduced. We observe also a small reduction of reassignment hit ratio from terrestrial to satellite path, which corresponding to situation where the gain of the reallocation is very low. However, this mechanism does not significantly affect the average flow completion time. A real implementation of an relocation procedure requiring a synchronization protocol, with a inherent overhead, the FlightTime mechanism is still attractive to reduce reassignments and related overheads.

\section{CONCLUSION}

In this paper, flow-based and packet-based routing mechanisms in a size-aware context have been compared. We, first, eliminate path aggregation as it degrades the Flow Completion Time due to an overuse of terrestrial path which is beneficial for short flows. Then, we propose differents packet-based mechanisms to improve the behavior of flow-based routing. These proposals do not show any notable improvement apart from few specific cases. Finally, studied flow-based routing give good results without the need for finer scale routing mechanisms in the size-aware considered context.

In our ongoing works, we are testing these routing schemes in a realistic environment with a reliable transport protocol. We are considering differents usage of the TCP transport protocol: a TCP sub-flows per path and per content, a pool of pre-established TCP flows, etc. Proposed improvement mechanisms, like Idle Link Opportunistic Aggregation or Reassignment, need inventive operation to be implemented efficiently. As our results show the low interest in packet-based algorithms, overcoming these implementation difficulties does no seem to be worth it. However, specific advantageous situation like low traffic condition could be identified in order to use specific appropriate mechanisms. Finally, our mechanisms will also be compared to MP-TCP which stands as the new reference for aggregation especially in heterogeneous networks.

\section{REFERENCES}

[1] N. M. Piratla and A. P. Jayasumana, "Reordering of packets due to multipath forwarding - an analysis," in 2006 IEEE International Conference on Communications, vol. 2, June 2006, pp. 829-834.

[2] K. Chebrolu and R. R. Rao, "Bandwidth aggregation for real-time applications in heterogeneous wireless networks," IEEE Transactions on Mobile Computing, vol. 5, no. 4, pp. 388-403, April 2006.

[3] C. Lai, K. C. Leung, and V. O. K. Li, "Does it hurt when others prosper?: Exploring the impact of heterogeneous reordering robustness of tcp," in 2013 Proceedings IEEE INFOCOM, April 2013, pp. 29582966.

[4] C. Paasch, S. Barre et al., "Multipath tcp in the linux kernel." [Online]. Available: http://www.multipath-tcp.org

[5] E. Bouttier, R. Dhaou, F. Arnal, C. Baudoin, E. Dubois, and A. L. Beylot, "Analysis of content size based routing schemes in hybrid satellite and terrestrial networks," in 2016 IEEE Global Communications Conference (GLOBECOM), Dec 2016, pp. 1-6.

[6] “CDN Interconnection Metadata," European Telecommunications Standards Institute, Tech. Rep., Mar. 2015.

[7] "Content delivery networks interconnection (cdni) working group." [Online]. Available: https://datatracker.ietf.org/wg/cdni/charter/

[8] B. Niven-Jenkins, R. Murray, M. Caulfield, and K. J. Ma, "CDN Interconnection Metadata," Internet Engineering Task Force, InternetDraft draft-ietf-cdni-metadata-13, Mar. 2016, work in Progress.

[9] D. R. Smith, "Technical note-a new proof of the optimality of the shortest remaining processing time discipline," Operations Research, vol. 26, no. 1, pp. 197-199, 1978. [Online]. Available: http://dx.doi.org/10.1287/opre.26.1.197

[10] J. Nagle, "On packet switches with infinite storage," IEEE Transactions on Communications, vol. 35, no. 4, pp. 435-438, Apr 1987.

[11] A. Demers, S. Keshav, and S. Shenker, "Analysis and simulation of a fair queueing algorithm," in ACM SIGCOMM Computer Communication Review, vol. 19, no. 4. ACM, 1989, pp. 1-12.

[12] A. Jain, A. Terzis, N. Sprecher, S. Arunachalam, K. Smith, H. Flinck, V. Devarapalli, and R. B. Yanai, "Mobile Throughput Guidance Inband Signaling Protocol," Internet Engineering Task Force, InternetDraft draft-flinck-mobile-throughput-guidance-04, Mar. 2017, work in Progress. [Online]. Available: https://datatracker.ietf.org/doc/html/draftflinck-mobile-throughput-guidance-04

[13] M. Leech, "Socks protocol version 5," RFC 4960 (Proposed Standard), Tech. Rep., 1996. 